\title{
Observations of plasma vortices in the vicinity of flow-braking:
} a case study

\author{
K. Keika ${ }^{1,2}$, R. Nakamura ${ }^{1}$, M. Volwerk ${ }^{1}$, V. Angelopoulos ${ }^{3}$, W. Baumjohann ${ }^{1}$, A. Retinò ${ }^{1}$, M. Fujimoto ${ }^{4}$, \\ J. W. Bonnell ${ }^{5}$, H. J. Singer ${ }^{6}$, H. U. Auster ${ }^{7}$, J. P. McFadden ${ }^{5}$, D. Larson ${ }^{5}$, and I. Mann ${ }^{8}$ \\ ${ }^{1}$ Space Research Institute, Austrian Academy of Sciences, Schmiedlstrasse 6, 8042 Graz, Austria \\ ${ }^{2}$ Center for Solar-Terrestrial Research, New Jersey Institute of Technology, 323 Martin-Luther-King Jr. Blvd., 101 Tiernan \\ Hall, Newark, NJ 07102-1982, USA \\ ${ }^{3}$ Institute of Geophysics and Planetary Physics, University of California, Los Angeles, CA 90095, USA \\ ${ }^{4}$ Institute of Space and Astronautical Science, Japan Aerospace Exploration Agency, Yoshinodai 3-1-1, Sagamihara \\ 229-8510, Japan \\ ${ }^{5}$ Space Science Laboratory, University of California, 7 Gauss Way \#7450, Berkeley, CA 94720-7450, USA \\ ${ }^{6}$ Space Weather Prediction Center, National Oceanic and Atmospheric Administration, 325 Broadway, Boulder, \\ CO 80305, USA \\ ${ }^{7}$ Institut für Geophysik und extraterrestrische Physik, Technische Universität Braunschweig, Mendelssohnstr. 3, \\ 38106 Braunschweig, Germany \\ ${ }^{8}$ Department of Physics, University of Alberta, 11322 - 89 Avenue, T6G 2G7 Edmonton, Alberta, Canada
}

Received: 21 October 2008 - Revised: 15 May 2009 - Accepted: 15 May 2009 - Published: 4 August 2009

\begin{abstract}
We examine fast plasma flows and magnetic field fluctuations observed by THEMIS at 03:00-03:30 UT on 12 December 2007. All THEMIS probes are situated in the near-Earth plasma sheet $\left(X_{S M}>-10 R_{E}\right)$ with $1-2 R_{E}$ spacecraft separations in azimuthal and radial directions. We focus on the observations of plasma convective flows made simultaneously by more than one THEMIS probe. At about 03:10 UT and 03:14 UT, the THEMIS P2 probe observed earthward flows of $>100 \mathrm{~km} / \mathrm{s}$. The THEMIS P1 probe, located duskward and earthward of P2, observed tailward flows under a positive $B_{z}$. The inner most probe THEMIS P4, located at almost the same MLT as THEMIS P1 and P2, did not see any clear flow. We examine the convective flow patterns for the THEMIS observations. We conclude that plasma vortices are formed near the region where the earthward flows slow down and turn in azimuthal directions.
\end{abstract}

Keywords. Magnetospheric physics (Magnetotail; Plasma convection; Plasma sheet)

\section{Correspondence to: K. Keika}

(keika@adm.njit.edu)

\section{Introduction}

In-situ plasma and magnetic field observations have revealed that earthward plasma transport in the central plasma sheet occurs with high speeds ( $>$ few $100 \mathrm{~km} / \mathrm{s}$ ) over short durations $(<$ few minutes) rather than as slow and long-duration convective flow (e.g., Baumjohann et al., 1990; Angelopoulos et al., 1992; Sergeev et al., 1992). Such fast earthward plasma flows, called bursty bulk flows, are interpreted as a consequence of reconnection that happened in the plasma sheet tailward of a spacecraft (e.g., Baumjohann, 2002).

When fast tailward flows are observed in the magnetotail, many are accompanied by a negative $B_{z}$, and interpreted as resulting from reconnection that occurred earthward of a spacecraft. It has been also reported that some tailward flows are seen when $B_{z}$ is positive. One explanation for these tailward flows is that a spacecraft is observing a traveling compression region (TCR) at the leading edge of tailward propagating structures, or plasmoids, generated through the reconnection (e.g., Slavin et al., 1984).

However, some of the tailward flows with a positive $B_{z}$ cannot be explained by the TCR concept, particularly when they are observed in the near-Earth magnetotail where reconnection rarely occurs. They are probably related to tailward moving closed flux tubes. Statistical studies by Schödel et

Published by Copernicus Publications on behalf of the European Geosciences Union. 



Fig. 1. Positions of THEMIS P1 to P5 and GOES 10 between 02:20 UT and 04:00 UT on 12 December 2007 in the XY (left) and $\mathrm{YZ}$ (right) planes in SM coordinates.

al. (2001a, b) showed that the probability of observing such tailward flows increases toward the Earth and that the flows are associated with dipolarizations. Their origin is, however, yet to be identified.

Three interpretations of the tailward flows have been proposed: rebounding of a closed flux tube due to an overcompression of the magnetic field (a pile-up effect) during the slowing down of earthward flow (Lopez, 1994; Chen and Wolf, 1999; Ohtani et al., 2009), flow shear around bubbles moving earthward or diverging around the dipole-like magnetic field (Chen and Wolf, 1993; Sergeev et al., 1996; Walsh et al., 2009), and vortices in the near-Earth plasma sheet (Fairfield et al., 1998). Numerical MHD simulations by Birn et al. (2004) demonstrated that a void tailward of earthward propagating depleted flux tubes is filled with mostly field-aligned flow. In addition, they showed perpendicular (convective) tailward flow behind the void, which is associated with the dawnward electric field.

In this paper, we report on multi-point observations of plasma convective flows in the near-Earth plasma sheet, some of which are tailward flows accompanied by a positive $B_{z}$. So far very few multi-point observations have been available in the regions earthward of a reconnection site and tailward of where earthward flow braking occurs (e.g., Shiokawa et al., 1997; Birn et al., 1999). A recently launched mission, the Time History of Events and Macroscale Interactions During Substorms (THEMIS) (Angelopoulos, 2008) which has five identical probes, provides an excellent opportunity to examine global as well as mesoscale dynamics of the near-Earth magnetotail depending on the spacecraft configuration. On 12 December 2007, all five probes observe magnetic field fluctuations and fast flows when they are situated in the near-Earth plasma sheet with $1-2 R_{E}$ spacecraft separations. A tailward flow with positive $B_{z}$ is observed by one probe at the same time an earthward flow is seen by another probe. We examine flow patterns during the simultaneous observations of the earthward and tailward flows. We conclude that plasma vortices are formed near the region where the fast earthward flow slows down and turns in an azimuthal direction.

\section{Dataset}

The present study uses the following data from THEMIS: 3 -s spin-fit magnetic field data obtained by the Fluxgate Magnetometer (FGM) (Auster et al., 2008), 3-s spin-fit electric field data obtained by the Electric Field Instrument (EFI) (Bonnell et al., 2008), ion data with energies of $\sim 5 \mathrm{eV}-25 \mathrm{keV}$ obtained by the Electro-Static Analyzer (ESA) plasma instrument (McFadden et al., 2008), and energetic ion data with energies above $\sim 30 \mathrm{keV}$ obtained by the Solid State Telescope (SST) (Larson et al., 2008).

We calculate the electric field component parallel to the spacecraft spin axis from the electric field perpendicular to the axis $\left(\boldsymbol{E}^{\prime}\right)$ and the magnetic field data $(\boldsymbol{B})$ when the angle between $\boldsymbol{E}^{\prime}$ and $\boldsymbol{B}$ is larger than $10^{\circ}$, assuming that $\boldsymbol{E}^{\prime} \times \boldsymbol{B}=$ zero. The electric field data is averaged over three data points in this study to provide $9 \mathrm{~s}$ samples. Using the reconstructed electric field $(\boldsymbol{E})$ with three components, we calculate $\boldsymbol{E} \times \boldsymbol{B}$ velocity.

ESA sweeps out $4 \pi$ steradians every spin period ( $3 \mathrm{~s}$ ) and provides 3 -s ion density and velocity data. Here we use the reduced distribution, in which the angular distribution is reduced from 88 to 50 angles. SST covers $4 \pi$ steradians every spin period ( $3 \mathrm{~s})$ and provides 3 -s ion density and velocity data. Angular resolutions for SST are $25^{\circ}$ in the azimuthal direction and $45^{\circ}$ in the polar direction in spacecraft coordinates. For ion velocity we use "joint velocity" data, where both ESA and SST data are combined; the joint velocity is calculated as: $\left(n_{\mathrm{ESA}} \boldsymbol{v}_{\mathrm{ESA}}+n_{\mathrm{SST}} \boldsymbol{v}_{\mathrm{SST}}\right) /\left(n_{\mathrm{ESA}}+n_{\mathrm{SST}}\right)$, where $n_{\mathrm{ESA}}$ and $\boldsymbol{v}_{\mathrm{ESA}}$ are density and velocity obtained by ESA, and $n_{\text {SST }}$ and $v_{\text {SST }}$ are density and velocity obtained by SST.

We also used the $0.512 \mathrm{~s}$ magnetic field data from GOES 10.

\section{December 2007 event observations}

\subsection{Overview}

Figure 1 shows positions of THEMIS and GOES 10 between 02:20 UT and 04:00 UT. The left and right panels represent the $X-Y$ and $Y-Z$ plane, respectively, in Solar Magnetic (SM) coordinates. All THEMIS probes were on their outbound paths and situated in the near-Earth magnetotail near the equator in SM coordinates. GOES 10 was in premidnight near the equator in SM coordinates.

Figure 2 shows an overview of GOES and THEMIS observations at 02:20-04:00 UT. Figure $2 \mathrm{a}$ to $\mathrm{f}$ presents observations by THEMIS P1, THEMIS P5, THEMIS P2, GOES 10, THEMIS P4, and THEMIS P3. Figure $2 \mathrm{a}, \mathrm{c}$, e, and $\mathrm{f}$ includes time series of the magnetic field, $\boldsymbol{E} \times \boldsymbol{B}$ velocity calculated from the electric field and magnetic field data, joint ion velocity perpendicular to the magnetic field, and ion energytime $(E-t)$ diagrams. Figure $2 \mathrm{~b}$ and $\mathrm{d}$ shows only magnetic field observations, because electric field and plasma 
(a)

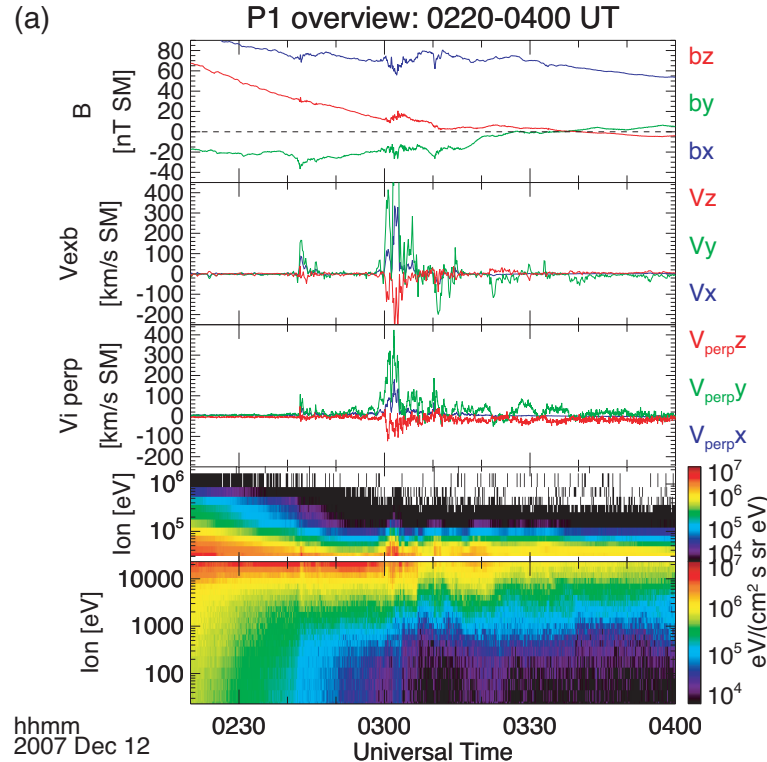

(b)



(c)

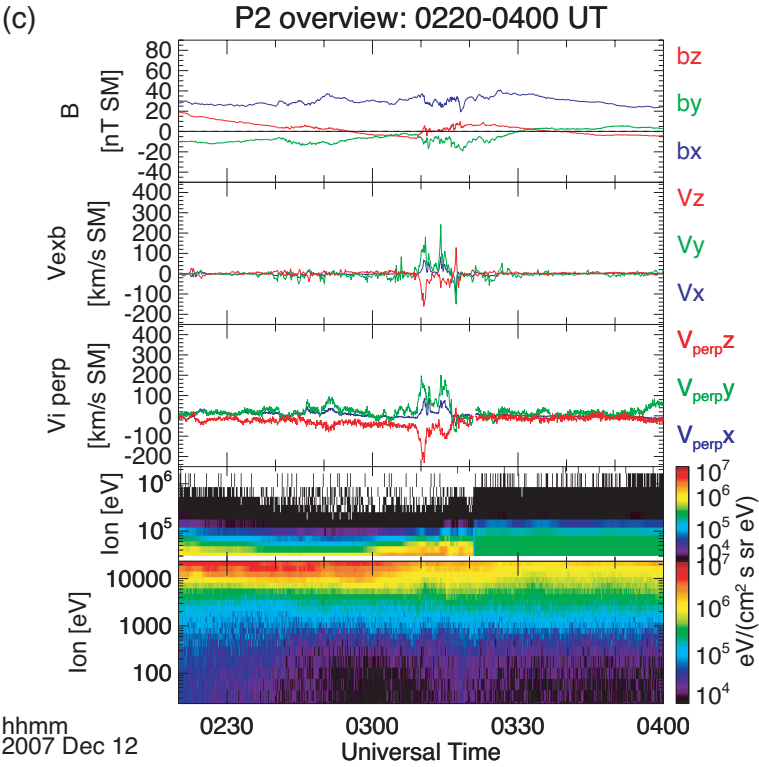

(d)

$\infty$

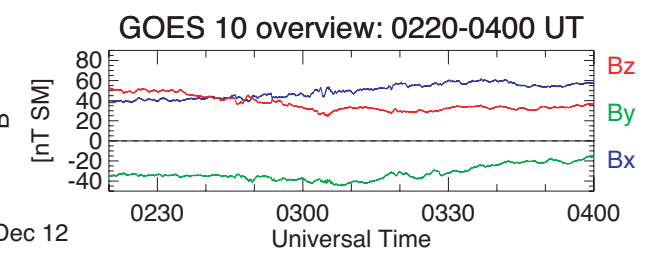

(e)

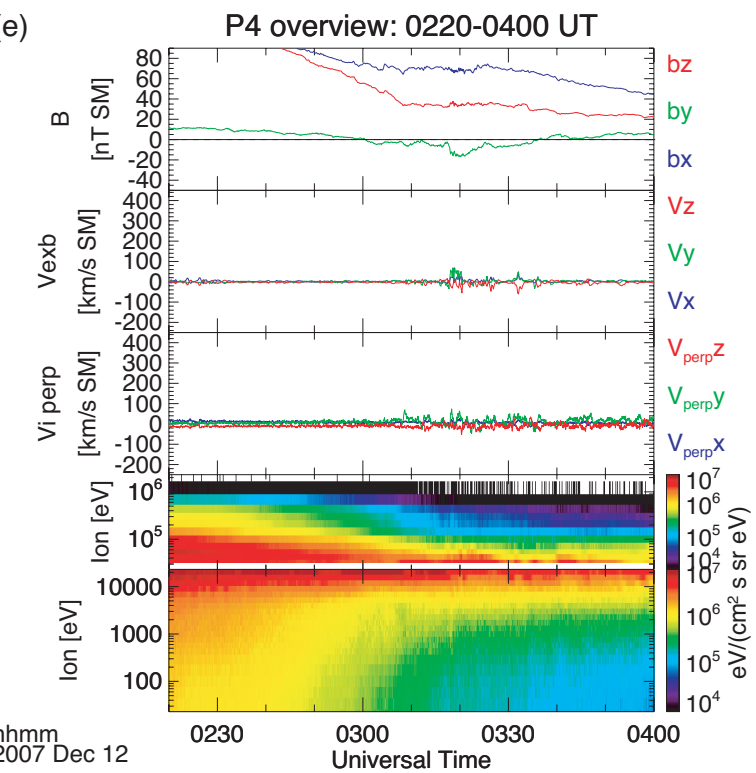

(f)

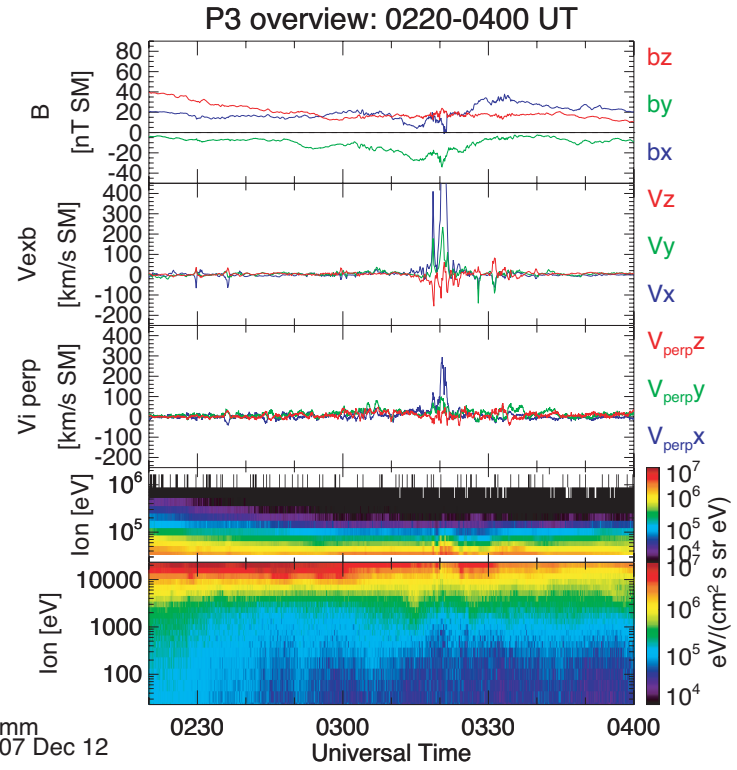

Fig. 2. An overview of THEMIS and GOES 10 observations and geomagnetic field variations at 02:20-04:00 UT on 12 December 2007. Panels for (a) THEMIS P1, (c) P2, (f) P3, and (e) P4 include the magnetic field, $\boldsymbol{E} \times \boldsymbol{B}$ velocity, joint ion velocity (obtained from both ESA and SST instruments), and ion $E-t$ diagrams. Panels for (b) THEMIS P5 and (d) GOES 10 show magnetic field observations.

data were not available for THEMIS P5 and GOES 10 does not make these measurements.

All probes observed fluctuations of the magnetic field between 03:00 UT and 03:30 UT. THEMIS P1 saw a dipo- larization and fast $(\sim 400 \mathrm{~km} / \mathrm{s})$ earthward and duskward flow at $\sim$ 03:00 UT (Fig. 2a). It also observed tailward and dawnward $\boldsymbol{E} \times \boldsymbol{B}$ flow with speed of $\sim 200 \mathrm{~km} / \mathrm{s}$ at 03:10 UT. Ion velocity derived from ion measurements was directed 
Magnetic Field \& ExB Velocity 0255-0330 UT

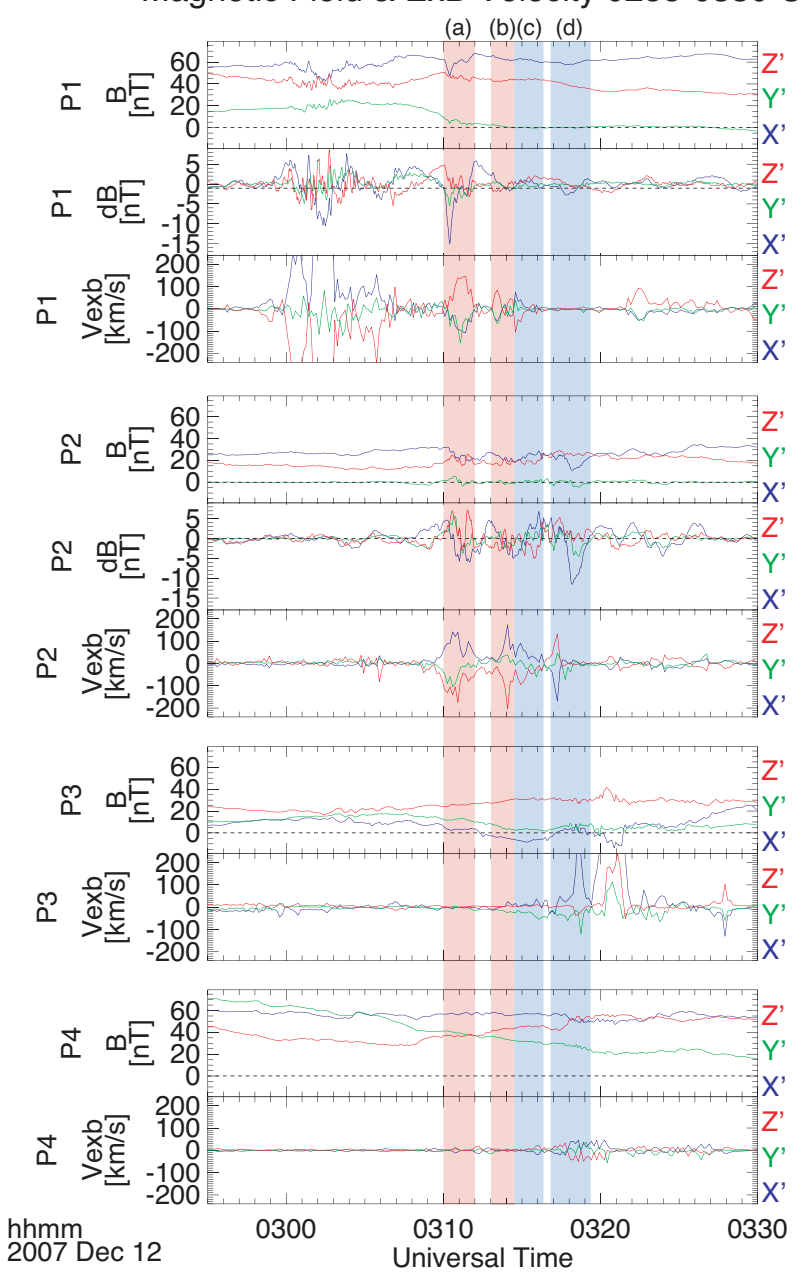

Fig. 3. The magnetic field and $\boldsymbol{E} \times \boldsymbol{B}$ velocity obtained in the new coordinate system (see Sect. 3.2). For P1 and P2, the deviations from the magnetic field smoothed with a 5-min average window are presented. Red and blue shadows indicate activations (a-d) during which more than one probe observed fast $\boldsymbol{E} \times \boldsymbol{B}$ flows.

duskward. Since the accurate calibration of SST data is yet to be completed, we cannot identify so far why $\boldsymbol{E} \times \boldsymbol{B}$ velocity and ion velocity disagree. (If this disagreement is due to physical phenomena, the radial pressure gradient and/or gradient $\boldsymbol{B}$ and curvature drifts of energetic ions are plausible candidates. They could modify the distribution function and therefore the velocity moments.)

THEMIS P2 saw a dipolarization and associated fast $(\sim 200 \mathrm{~km} / \mathrm{s})$ earthward and duskward $\boldsymbol{E} \times \boldsymbol{B}$ flow at $\sim 03: 10$ UT (Fig. 2c). It also observed tailward and dawnward $\boldsymbol{E} \times \boldsymbol{B}$ flow $(\sim 100 \mathrm{~km} / \mathrm{s})$ at $\sim 03: 18$ UT. (A sudden change in energetic ion flux shown in the $E-t$ diagram is related to the SST particle attenuator opening. The SST attenuator is used in the inner magnetosphere for reducing large fluxes of radiation. The SST accurate calibrations are not completed and this causes lack of removal of the background.) THEMIS P3 observed a fast earthward $\boldsymbol{E} \times \boldsymbol{B}$ flow ( $>300 \mathrm{~km} / \mathrm{s}$ ) at $\sim 03: 20$ UT (Fig. 2f). THEMIS P4 observed slight fluctuations of plasma flows, while it saw no clear earthward fast flow during the whole interval (Fig. 2e). THEMIS P5 observed a dipolarization at $\sim 03: 10$ UT and GOES 10 saw a small dipolarization at $\sim 03: 05$ UT.

During the first half of the interval shown in Fig. 2, $B_{y}$ was negative and sometimes comparable to $B_{x}$ or $B_{z}$ at THEMIS P1, THEMIS P2, THEMIS P3, and GOES 10, even though the probes, except for GOES 10, were around midnight or on the dawn side where $B_{y}$ is usually nearly zero or positive as seen in various magnetic field models such as Tsyganenko's models (e.g., Tsyganenko, 2002). The probes are located closer to the magnetic equator and further away from the Earth than THEMIS P4 and P5 (see Fig. 1). The observations suggest a strongly tilted plasma sheet which is rotated clockwise around the $\mathrm{X}$ axis or anticlockwise around the Z-axis.

This negative $B_{y}$ started at about 01:40 UT (not shown here) and remains until 03:30 UT. According to Geotail solar wind observations upstream of the bow shock $\left(X \sim 29 R_{E}\right)$, the interplanetary field (IMF) was dominated by negative $B_{y}$ until 01:50 UT at the Geotail location (not shown here). Since solar wind speed exceeds $600 \mathrm{~km} / \mathrm{s}$ during the interval, the negative IMF $B_{y}$ would have passed by the magnetotail within $30 \mathrm{~min}$, by $\sim 02: 20 \mathrm{UT}$. Therefore, the dominant negative IMF $B_{y}$ alone does not seem to be responsible for the tilted plasma sheet. We have not identified what leads to the unusual plasma sheet configuration. The present study focuses on magnetic field fluctuations and fast plasma flow at 02:55-03:30 UT.

\subsection{Magnetic field fluctuations and fast flows}

As introduced in Sect. 3.1, $B_{y}$ is large and unexpectedly negative during the interval of 02:55-03:30 UT. Since it is easier to examine fast earthward flow- and dipolarizationassociated magnetic field fluctuations in the $X-Z$ plane, we change the coordinate system in which the observed magnetic field variations are in only two components (i.e., new $\boldsymbol{X}^{\prime}$ and $\boldsymbol{Z}^{\prime}$ components). The coordinate transformation also makes it easer to examine a flow pattern in the plane parallel to the current sheet (plasma sheet), because a large background dawn-dusk component (i.e., $B_{y}$ ) disappears.

The new coordinate system that we chose is $\left(\boldsymbol{X}^{\prime}, \boldsymbol{Y}^{\prime}, \boldsymbol{Z}^{\prime}\right)=(\boldsymbol{r}$, $\boldsymbol{b} \times \boldsymbol{r}, \boldsymbol{r} \times(\boldsymbol{b} \times \boldsymbol{r})$ ), where $\boldsymbol{r}$ is a unit vector from a THEMIS P2 position at 03:10 UT toward the Earth and $\boldsymbol{b}$ is a unit vector of the magnetic field observed by THEMIS P2. $\boldsymbol{b}$ is smoothed with a 5 min window running average which is a little longer than the time scale of the fast plasma flows $(\sim 2 \mathrm{~min})$ observed by THEMIS during the interval of interest. We apply the new coordinate system to data from the other probes. The reason why we use THEMIS P2 data to define the coordinate system is that THEMIS P2 observed in most cases 
fast earthward flows and we are studying how the earthward flows evolve in the vicinity of flow braking. Figure 3 shows magnetic field and $\boldsymbol{E} \times \boldsymbol{B}$ velocity data in the new coordinate system for THEMIS P1, P2, P3, and P4 from top down. In the first and second panels, the deviations from the magnetic field smoothed with a 5-min average window are also presented.

In this paper, we study plasma convective flows observed simultaneously by more than one THEMIS probe. During the four intervals (a)-(d) indicated by red and blue shadows in Fig. 3, fast $\boldsymbol{E} \times \boldsymbol{B}$ flows were observed by more than one THEMIS probe.

$\boldsymbol{E} \times \boldsymbol{B}$ flows were tailward and dawnward at THEMIS P1 and nearly earthward at THEMIS P2 during the interval (a). The observations indicate a plasma vortex (see Sect. 3.3 in details). The magnetic field changed by $5-15 \mathrm{nT}$. At THEMIS P2 observing the earthward flow, the $\boldsymbol{Z}^{\prime}$ component increased and the $\boldsymbol{X}^{\prime}$ component decreased with fluctuations. The $\boldsymbol{X}^{\prime}$ and $\boldsymbol{Z}^{\prime}$ components at THEMIS P1, on the other hand, decreased. The interval (b) also shows indications of a plasma vortex; $\boldsymbol{E} \times \boldsymbol{B}$ flows were tailward and dawnward at THEMIS P1 and nearly earthward at THEMIS P2. The magnetic field $\boldsymbol{X}^{\prime}$ component decreased at both probes, and the $\boldsymbol{Z}^{\prime}$ component was not greatly changed. Such magnetic field variations in the $\boldsymbol{X}^{\prime}$ and $\boldsymbol{Z}^{\prime}$ components are similar to those expected at the time of dipolarization or plasma sheet expansion. (It should be noted that the large negative $B_{y}$ at THEMIS P2 shown in Fig. 2 disappears and magnetic field fluctuations are dominated by the $\boldsymbol{X}^{\prime}$ and $\boldsymbol{Z}^{\prime}$ components. In addition, $B_{y^{\prime}}$ at THEMIS P1 becomes close to zero after 03:10 UT. Thus, we can discuss the field variations in a background magnetic field dominated by the nearly sunward and northward components.) In addition, we expected that the fluctuations also resulted from field-aligned currents (FACs) due to field twists or shear lead by the plasma vortex. We discuss causes of the field fluctuations in Sect. 4.

At the interval (c), THEMIS P1, P2, and P3 observed convective flow with different directions. THEMIS P4 saw no clear earthward nor tailward flow during the three intervals. At the interval (d), THEMIS P3 saw earthward fast flow, while THEMIS P2 observed tailward flow. THEMIS P4 saw relatively slow $\boldsymbol{E} \times \boldsymbol{B}$ flow. The observations imply flow divergence around the dipole-like magnetic field (see Sect. 3.3 in details).

\subsection{Flow patterns}

In this section, we examine flow patterns in the four intervals (a)-(d). We use $\boldsymbol{E} \times \boldsymbol{B}$ flow data because we focus on convective plasma flows. Figure 4 shows flow patterns in the $X^{\prime}-Y^{\prime}$ (left panel), $Y^{\prime}-Z^{\prime}$ (right panel) and $X^{\prime}-Z^{\prime}$ planes at the intervals (a)-(d) from top down. Diamonds in each panel represent probe positions. Thin arrows indicate $\boldsymbol{E} \times \boldsymbol{B}$ flow direction and their colors correspond to probe numbers. Thick black arrows show averaged flow direction and strength. " $\mathrm{X}$ " signs on the diamonds denote no clear flow observed by the corresponding probe.

Flow patterns shown in Fig. 4a and b suggest a plasma vortex at the time of earthward fast flow. Since the inner most probe (P4) saw no clear flow, we suggest that the plasma vortex is positioned near the region where earthward flow braking occurs (flow-braking region). In fact, the magnetic field at P4 is more dipole-like than that at P1 and P2 (see Fig. 3). P3 also observed no clear flow during the interval. We believe that this is because P3 was azimuthally distant from the main earthward flow channel and/or inward of the flow-braking region. Figure 5 illustrates a flow vortex pattern (dashed arrows and lines) in the $X^{\prime}-Y^{\prime}$ and $X^{\prime}-Z^{\prime}$ planes along with spacecraft positions (diamonds) and the observed flow directions (solid arrows). Big dashed arrows represent the source earthward flow, and small dashed arrows show plasma flows near the flow-braking region. Solid curved lines in the $X^{\prime}-Z^{\prime}$ plane panel illustrate stretched and dipole-like magnetic fields.

Figure $4 \mathrm{c}$ indicates that the plasma flow is braking and diverging around the dipole-like magnetic field. Flow patterns in Fig. 4d are not clear; flow at P2 and P3 are fluctuating more than during the other intervals. Although it is not so easy to explain this pattern, THEMIS P2 and P3 might see both plasma vortex and flow divergence near the flow-braking region. We believe that flow at P4 is diverging around the dipole-like magnetic field.

\section{Summary and discussion}

We examined fast plasma flows and magnetic field fluctuations, including dipolarizations, observed by THEMIS at 03:00-03:30 UT on 12 December 2007. All THEMIS probes were situated in the near-Earth plasma sheet. They provided simultaneous observations of plasma convective flows made by two or three probes separated by $1-2 R_{E}$ in azimuthal and radial directions (see Fig. 1). We studied plasma flow patterns for the observations by using a new coordinate system in which the field fluctuations associated with the earthward flows are in only two components. Flow patterns during two of the four examined intervals ( $\sim 03: 10$ UT and $\sim 03: 14$ UT) showed that the plasma flows at THEMIS P1 were almost in a direction opposite to the earthward-dominant flows at THEMIS P2 azimuthally separated from THEMIS P1. No clear flow was observed by the inner most probe THEMIS P4 which was located in almost the same MLT as THEMIS P2 (Fig. 4a and b). We conclude that plasma vortices are formed in the vicinity of the flow braking, within a $1-2 R_{E}$ distance from the earthward edge of the flow channel of earthward flows (i.e., where earthward flows slow down and turn in azimuthal directions). An illustration of the plasma vortex along with the flow observations is drawn in Fig. 5. The braking of earthward flows and its diverging around the 
(a) 2007/12/12 0310:00 UT - 0312:00 UT •P1 $\bullet$ P2 $\bullet$ P3 $\bullet$ P4
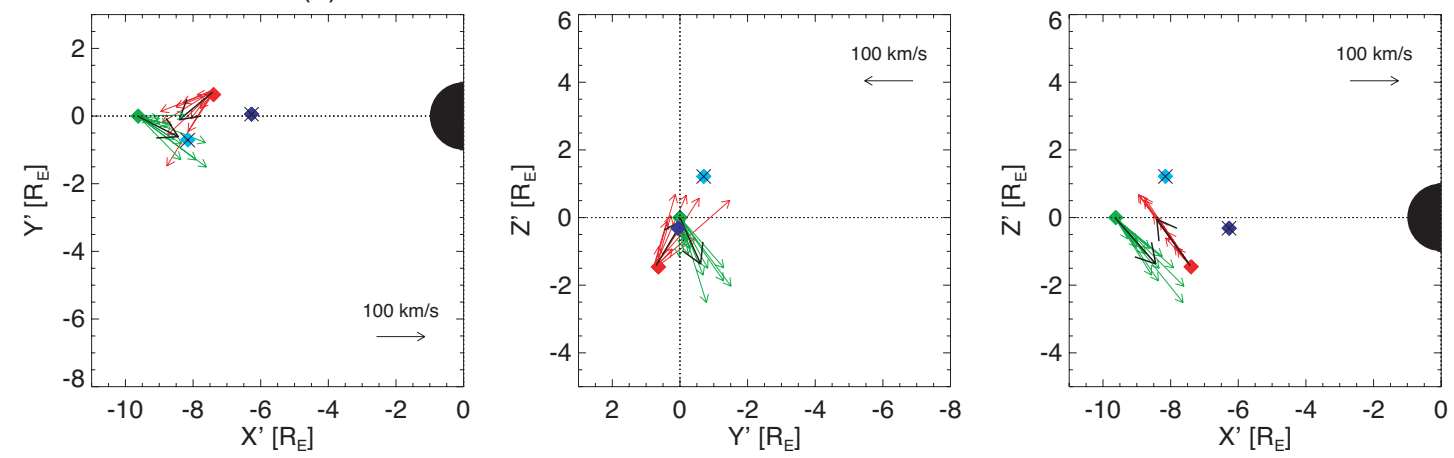

(b) $2007 / 12 / 12$
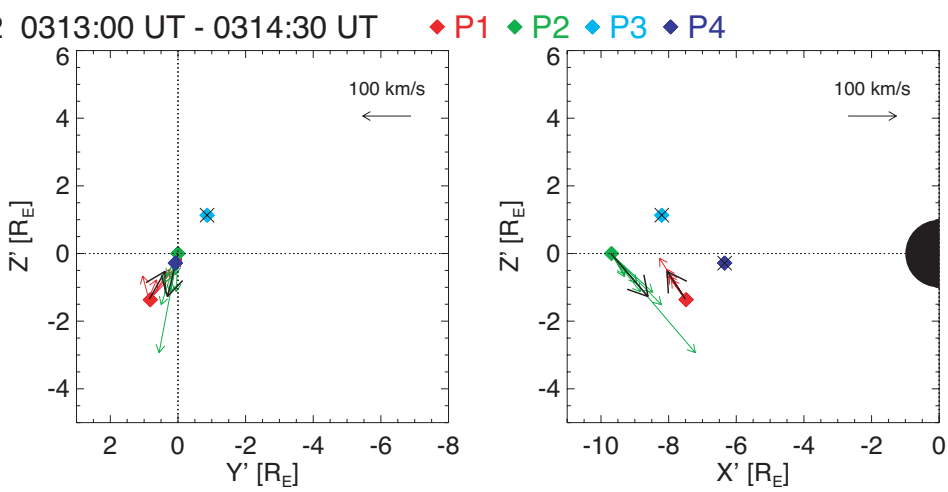

(c) $2007 / 12 / 12$
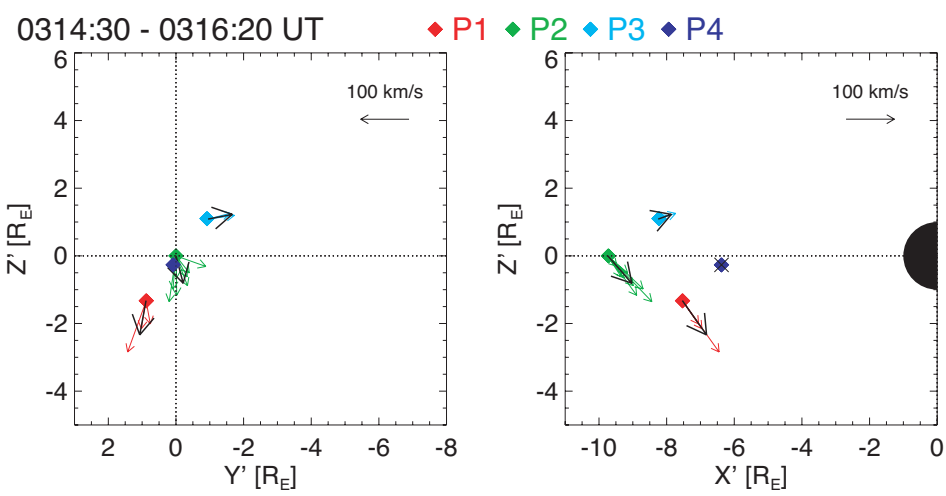

(d) $2007 / 12 / 12$
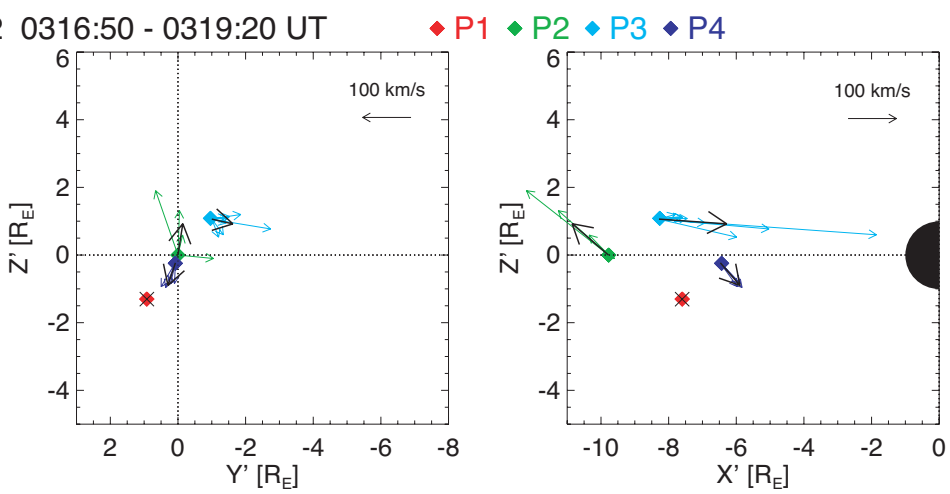

Fig. 4. Plasma flow patterns during the four intervals indicated by the red and blue shadows in Fig. 3 in the $X^{\prime}-Y^{\prime}$ (left), $Y^{\prime}-Z^{\prime}$ (right), and $X^{\prime}-Z^{\prime}$ planes of the new coordinate system. Diamonds represent spacecraft positions and thin arrows indicate flow direction and strength. Each thick arrow shows an average of fast flows indicated by the corresponding thin arrows. "X" signs denote no clear flow observed. 

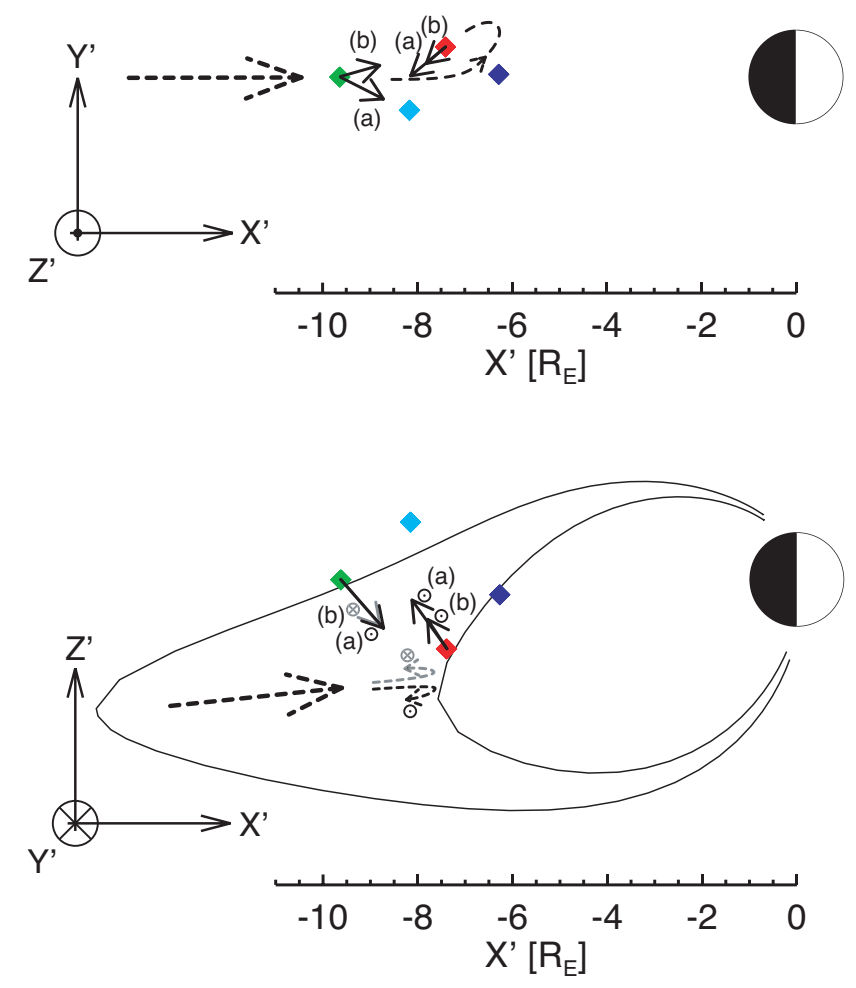

Fig. 5. Illustration of a flow vortex pattern in $X^{\prime}-Y^{\prime}$ and $X^{\prime}-Z^{\prime}$ planes. Big dashed arrows represent the source earthward flow. Small dashed arrows and lines illustrate plasma flows near the earthward edge of the earthward flow channel. Solid arrows indicate the directions of the observed flows for the intervals (a) and (b). The Y-components of the flows in the $X^{\prime}-Z^{\prime}$ plane are denoted by the marks adjacent to the arrows. Diamonds represent THEMIS positions. Solid curved lines in the $X^{\prime}-Z^{\prime}$ plane panel illustrate stretched and dipole-like magnetic fields.

dipole-like magnetic field were also seen during another interval (Fig. 4c).

The formation of the vortices may be initiated by shear flows ahead of an earthward moving flux tubes (e.g., Sergeev et al., 1996). However, such shear flows alone would have difficulties creating the flows directed toward the flow channel of the earthward moving flux tubes. We believe that existence of the earthward boundary of flow channels (where earthward flows turn in an azimuthal direction) resulting in divergence of the earthward flows around the dipole-like magnetic field plays an important role in generating vortices. In fact, the THEMIS observations examined in this paper showed that the tailward and dawnward flows (observed by THEMIS P1) directed toward the flow channel of the earthward flows (observed by THEMIS P2) appears near the region where the earthward flows slow down and turn in azimuthal directions. The observations for another interval indicate the divergence of earthward flows around the dipolelike magnetic field. (a) 0310:00 - 0312:00 UT

(b) 0313:00 - 0314:30 UT
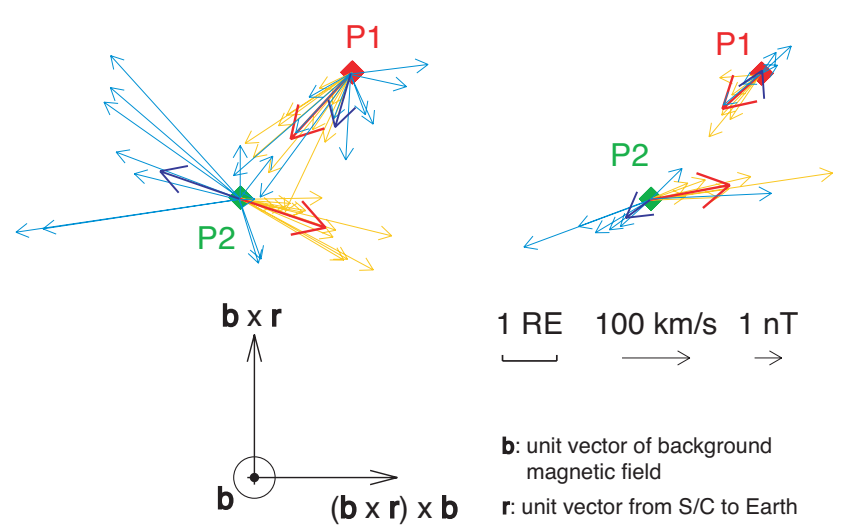

Fig. 6. Magnetic field variations (blue thin arrows) and plasma flow patterns (orange thin arrows) at (a) 03:10:00-03:12:00 UT and (b) $03: 13: 00-03: 14: 30$ UT in the plane perpendicular to the local magnetic field (see Sect. 4. for detailed descriptions of the coordinate system). Diamonds represent spacecraft positions. Red and blue thick arrows indicate the average of the corresponding plasma flows and magnetic field variations, respectively.

The observed flow patterns are not likely due to rebounding of the flux tubes transported earthward. The tailward (and dawnward) flows observed at THEMIS P1 were not preceded by earthward flows. If the tailward flows were due to the rebounding, the spacecraft would have seen any earthward flow prior to the tailward flows.

It has been suggested that a plasma vortex leads to a twist or shear of the magnetic field when MHD assumptions are satisfied (e.g., Birn et al., 2004). Magnetic field twists or shears lead by a pair of plasma vortices associated with fast earthward flow in the vicinity of flow braking corresponds to region-1 sense field-aligned currents (FACs) consistent with the formation of a substorm current wedge (e.g., Birn et al., 2004). Keiling et al. (2009) reported conjugate magnetospheric and ionospheric flow vortices during the formation of the substorm current wedge. They estimated the amplitude of the generated FACs, presenting that vortices generated FACs at the beginning of the substorm expansion phase. In order to examine the existence of FAC signatures in the magnetic field data of our event, we plot the magnetic field variations in the plane perpendicular to the magnetic field along with plasma flow patterns for the intervals (a) (03:10:00-03:12:00 UT) and (b) (03:13:00-03:14:30 UT) (see Sect. 3.3) as shown in Fig. 6. One axis in the perpendicular plane is in the direction of $\boldsymbol{b} \times \boldsymbol{r}$, where $\boldsymbol{b}$ is a unit vector of a 5-min window running average of the magnetic field and $\boldsymbol{r}$ is a unit vector from a spacecraft position to the Earth. The other axis is the cross product of $\boldsymbol{b} \times \boldsymbol{r}$ and $\boldsymbol{b}$, lying in the same plane as $\boldsymbol{b}$ and $\boldsymbol{r}$. The coordinate system is determined at each spacecraft, giving the plane perpendicular to the magnetic field to both spacecraft data. Relative spacecraft positions are presented in SM 
coordinates. Orange thin arrows indicate flow direction and strength (same as the right panels of Fig. $4 \mathrm{a}$ and $\mathrm{b}$ except for color). Red thick arrows present averaged direction and strength. Thin blue arrows indicate the magnetic field variations, which are defined as deviations from the 5-min window running average. Thick blue arrows show averages of the variations.

The variation averages are in a direction anti-parallel to the flows at THEMIS P2 (green diamond) for the interval (a) (Fig. 6a) and at THEMIS P1 (red diamond) in the interval (b) (Fig. 6b). The magnetic field direction is consistent with an expected direction of magnetic field changes due to FACs generated by a plasma vortex, because FACs generated by a vortex create the magnetic field anti-parallel to the vortex. The variations of the magnetic field are, however, more scattered than the plasma flow variations. The direction of the field variations at THEMIS P1 (red diamond) for the first interval (Fig. 6a) and at THEMIS P2 (green diamond) for the second interval (Fig. 6b) is inconsistent with a field direction due to FACs generated by a vortex. The FAC-related magnetic field variations were therefore present only a part of the observations.

The most plausible reason why it is difficult to identify FAC-related magnetic field signatures is that magnetic field fluctuations at the time of a fast flow and its braking contain the effects of the reconfiguration of the flux tubes (e.g., dipolarization) and the expansion of the plasma sheet as well as of FACs generated by a plasma vortex. $B_{z}$ increases and $B_{x}$ decreases at the dipolarization, and $B_{x}$ decreases when the plasma sheet becomes expanded. Such field variations are believed to be usually larger than those due to FACs. Both dipolarization and plasma sheet expansion can be frequently seen when a spacecraft observes an earthward fast flow.

Acknowledgements. THEMIS was made possible and is supported in the US by NASA NAS5-02099. The work of the IGEP team at the Technical University of Braunschweig was financially supported by the German Ministerium für Wirtschaft und Technologie and the German Zentrum für Luft- und Raumfahrt under grant 50QP0402.

Editor in Chief W. Kofman thanks one anonymous referee for her/his help in evaluating this paper.

\section{References}

Angelopoulos, V.: The THEMIS mission, Space Sci. Rev., 141, 534, doi:10.1007/s11214-11008-19336-11211, 2008.

Angelopoulos, V., Baumjohann, W., Kennel, C. F., Coronti, F. V., Kivelson, M. G., Pellat, R., Walker, R. J., Lühr, H., and Paschmann, G.: Bursty bulk flows in the inner central plasma sheet, J. Geophys. Res., 97, 4027-4039, 1992.

Auster, H. U., Glassmeier, K. H., Magnes, W., et al.: The THEMIS fluxgate magnetometer, Space Sci. Rev., 141, 235-264, doi10.1007/s11214-11008-19365-11219, 2008.

Baumjohann, W.: Modes of convection in the magnetotail, Phys. Plasma, 9, 3665-3667, 2002.
Baumjohann, W., Paschmann, G., and Lühr, H.: Characteristics of high-speed ion flows in the plasma sheet, J. Geophys. Res., 95, 3801-3809, 1990.

Birn, J., Hesse, M., Haerendel, G., Baumjohann, W., and Shiokawa, K.: Flow braking and the substorm current wedge, J. Geophys. Res., 104, 19895-19903, 1999.

Birn, J., Raeder, J., Wang, Y. L., Wolf, R. A., and Hesse, M.: On the propagation of bubbles in the geomagnetic tail, Ann. Geophys., 22, 1773-1786, 2004, http://www.ann-geophys.net/22/1773/2004/.

Bonnell, J. W., Mozer, F. S., Delory, G. T., Hull, A. J., Ergun, R. E., Cully, C. M., Angelopoulos, V., and Harvey, P. R.: The electric field instrument (EFI) for THEMIS, Space Sci. Rev., 141, 303341, doi:10.1007/s11214-008-0469-2, 2008.

Chen, C. X. and Wolf, R. A.: Interpretation of high-speed flows in the plasma sheet, J. Geophys. Res., 98, 21409-21419, 1993.

Chen, C. X. and Wolf, R. A.: Theory of thin-filament motion in Earth's magnetotail and its application to bursty bulk flows, J. Geophys. Res., 104, 14613-14626, 1999.

Fairfield, D. H., Mukai, T., Lui, A. T. Y., et al.: Geotail observations of substorm onset in the inner magnetotail, J. Geophys. Res., 103, 103-117, 1998.

Keiling, A., Angelopoulos, V., Runov, A., et al.: Substorm current wedge driven by plasma flow vortices: THEMIS observations, J. Geophys. Res., in press, doi:10.1029/2009JA014114, 2009.

Larson, D., Angelopoulos, A., and McFadden, J. P.: THEMIS SST instrument, Space Sci. Rev., in review, 2008.

Lopez, R. E., Goodrich, C. C., Reeves, G. D., Belian, R. D., and Taktakishvili, A.: Midtail plasma flows and the relationship to near-earth substorm activity: A case study, J. Geophys. Res., 99, 23561-23569, 1994.

McFadden, J. P., Carlson, C. W., Larson, D., Angelopoulos, V., Ludlam, M., Abiad, R., and Elliot, B.: The THEMIS ESA plasma instrument and in-flight calibration, Space Sci. Rev., 141, 277-302, doi:10.1007/s11214-008-9440-2, 2008.

Ohtani, S., Miyashita, Y., Singer, H., and Mukai, T.: Tailward Flows with Positive Bz in the Near-Earth Plasma Sheet, J. Geophys. Res., in press, doi:10.1029/2009JA014159, 2009.

Schödel, R., Baumjohann, W., Nakamura, R., Sergeev, V. A., and Mukai, T.: Rapid flux transport in the central plasma sheet, J. Geophys. Res., 106, 301-313, 2001a.

Schödel, R., Nakamura, R., Baumjohann, W., and Mukai, T.: Rapid flux transport and plasma sheet reconfiguration, J. Geophys. Res., 106, 8381-8390, 2001 b.

Sergeev, V. A., Elphic, R. C., Mozer, F. S., Saint-Marc, A., and Sauvaud, J. A.: A two-satellite study of nightside flux transfer events in the plasma sheet, Planet. Space Sci., 40, 1551-1572, 1992.

Sergeev, V. A., Angelopoulos, V., Gosling, J. T., Cattell, C. A., and Russell, C. T.: Detection of localized, plasma-depleted flux tubes or bubbles in the midtail plasma sheet, J. Geophys. Res., 101, 10817-10826, 1996.

Shiokawa, K., Baumjohann, W., and Haerendel, G.: Braking of high-speed flows in the near-earth tail, Geophys. Res. Lett., 24, 1179-1182, 1997.

Slavin, J. A., Smith, E. J., Tsurutani, B. T., Sibeck, D. G., Singer, H. J., Baker, D. N., Gosling, J. T., Hones, E. W., and Scarf, F. L.: Substorm associated traveling compression regions in the distant tail: ISEE-3 geotail observations, Geophys. Res. Lett., 11, 657- 
660, 1984.

Tsyganenko, N. A.: A model of the magnetosphere with a dawndusk asymmetry, 1, Mathematical structure, J. Geophys. Res., 107(A8), 1179, doi:10.1029/2001JA000219.
Walsh, A. P., Fazakerley, A. N., Lahiff, A. D., Volwerk, M., Grocott, A., Dunlop, M. W., Lui, A. T. Y., Kistler, L. M., Lester, M., Mouikis, C., Pu, Z., Shen, C., Shi, J., Taylor, M. G. G. T., Lucek, E., Zhang, T. L., and Dandouras, I.: Cluster and Double Star multipoint observations of a plasma bubble, Ann. Geophys., 27, 725-743, 2009, http://www.ann-geophys.net/27/725/2009/. 